\title{
Knockdown of PDIA6 Inhibits Cell Proliferation and Enhances the Chemosensitivity in Gastric Cancer Cells
}

This article was published in the following Dove Press journal: Cancer Management and Research

\author{
Chao Yan ${ }^{1, *}$ \\ Xiaolei Song ${ }^{2, *}$ \\ Su Wang ${ }^{3}$ \\ Jinhai Wang ${ }^{4}$ \\ $\mathrm{Lu} \mathrm{Li}^{4}$
}

'Department of Radiation Oncology, Qilu Hospital (Qingdao), Cheeloo College of Medicine, Shandong University, Qingdao 266035, People's Republic of China; ${ }^{2}$ Intervention Department, Qilu Hospital (Qingdao), Cheeloo College of Medicine, Shandong University, Qingdao 266035, People's Republic of China; ${ }^{3}$ Department of General Surgery, Qingdao Chengyang People's Hospital, Qingdao 266109, People's Republic of China; ${ }^{4}$ Gastroenterology Department, The Second Affiliated Hospital of Xi'an Jiaotong University, Xi'an 710004,

People's Republic of China

*These authors contributed equally to this work
Correspondence: Lu Li Gastroenterology Department, The Second Affiliated Hospital of Xi'an Jiaotong University, Xi'an 710004,

People's Republic of China

Tel/Fax +86-29-87679290

Email lilu050505@|26.com
Background: Protein disulfide isomerase A6 (PDIA6), a member of the disulfide isomerase (PDI) family, has been reported to be closely associated with progression of various cancers. However, the specific effects of PDIA6 on gastric cancer (GC) remain unclear. In this study, we investigated the expression pattern and biological functions of PDIA6 in GC.

Materials and Methods: The CCK- 8 assay was carried out to examine cell proliferation and cisplatin cytotoxicity. The Western blot analysis was used to measure the protein expression of PDIA6, Wnt3a and $\beta$-catenin. The xenograft tumor assay was performed to evaluate the in vivo effect of PDIA6 on GC cell proliferation and chemoresistance.

Results: PDIA6 was significantly elevated in GC tissues and cell lines. Down-regulation of PDIA6 inhibited GC cell proliferation and chemoresistance to cisplatin while up-regulation of PDIA6 promoted the proliferation and chemoresistance of GC cells. Besides, PDIA6 regulated the chemosensitivity of GC cells to cisplatin in vivo. Mechanically, PDIA6 served as a regulator of the $\mathrm{Wnt} / \beta$-catenin signaling pathway by affecting the protein expression of Wnt3a and $\beta$-catenin in GC cells. Additionally, Wnt activator reversed the inhibitory effect of PDIA6 knockdown on cisplatin resistance in GC cells.

Conclusion: These findings provided new insight into the potential role of PDIA6 as a promising target for drug resistance in GC chemotherapy.

Keywords: PDIA6, proliferation, chemosensitivity, gastric cancer

\section{Introduction}

Gastric cancer (GC), one of the most common and fatal malignancies, is the third leading cause of cancer-associated death in the world. ${ }^{1}$ With development of therapeutic approaches, the mortality of GC patients has fallen dramatically over the past decades. ${ }^{2,3}$ But, due to atypical symptoms at an early stage, a majority of GC patients are diagnosed at a late stage, resulting in an unfavorable prognosis. ${ }^{4}$ In this case, chemotherapy is considered a main therapeutic strategy to improve the average survival and quality of life in GC patients. ${ }^{5,6}$ However, chemoresistance, a major obstacle for cancer therapies, becomes common during treatment. ${ }^{7}$ Thus, it is urgently required to explore novel chemotherapeutic strategies for enhancement of chemosensitivity.

Protein disulphide isomerase (PDI), the first folding catalyst isolated from rat livers, abounds in many tissues. ${ }^{8}$ So far, 21 members have been identified in the PDI family and these proteins have different enzymatic properties. ${ }^{9,10}$ PDI could be induced under endoplasmic reticulum stress and is capable of isomerizing, forming 
and rearranging protein disulphide bonds. ${ }^{11-13}$ Besides, increasing evidence has demonstrated an important role of PDI in both the physiology and pathophysiology of disease states such as neurodegenerative conditions and the pathogen entry in infectious diseases. ${ }^{14,15}$ Recently, many studies have reported an association between the PDI family and cancer progression. For example, PDIA6, a member of the PDI family, was found serving as an oncogene in cervical cancer. ${ }^{16}$ In addition, PDIA6 was demonstrated to be a mediator of chemoresistance in lung adenocarcinoma. ${ }^{17}$ However, the specific effects of PDIA6 on GC remain unclear.

In this study, we investigated the biological functions of PDIA6 in GC. We observed that PDIA6 was overexpressed in GC tissues and cell lines. Knockdown of PDIA6 inhibited GC cell proliferation in vitro and in vivo. In addition, we found that PDIA6 could affect chemoresistance in GC cells partly via the Wnt/ $\beta$-catenin pathway.

\section{Materials and Methods}

\section{Patients and Tissue Samples}

A total of 48 patients from Qilu Hospital, Cheeloo College of Medicine (Qingdao, China) were enrolled in this study. All patients received no preoperative therapies and provided written informed consent. GC tissues and adjacent normal tissues were collected and frozen in liquid nitrogen and stored at $-80^{\circ} \mathrm{C}$. The study was approved by the Ethics Committee of Qilu Hospital (Qingdao), Cheeloo College of Medicine.

\section{Cell Lines and Cell Culture}

GC cell lines (BGC-823 and MKN-45) and normal gastric epithelial cell line GES-1 were purchased from American Type Culture Collection (ATCC, Manassas, VA, USA). All cell lines were cultured in RPMI-1640 medium (Invitrogen, Carlsbad, CA, USA) supplemented with $10 \%$ fetal bovine serum (FBS; Invitrogen) and $100 \mathrm{U} / \mathrm{mL}$ penicillin/streptomycin (Invitrogen) at $37^{\circ} \mathrm{C}$ in a humidified atmosphere with $5 \% \mathrm{CO}_{2}$.

\section{Quantitative Real-Time Polymerase Chain} Reaction (RT-qPCR)

Total RNA was isolated from tissues or cells using the Trizol reagent (Thermo Fisher Scientific, Waltham, MA, USA). Reverse transcription was performed using PrimeScript ${ }^{\mathrm{TM}}$ RT Reagent Kit (Takara Biotechnology, Dalian, China). The following primers were used: PDIA6, 5'-
TGGATCCAACAAAAACAGACC-3' (forward) and 5'CTCAGCGCAGCATCTACAAT-3' (reverse); GAPDH, 5'ACCACAGTCCATGCCATCAC-3' (forward) and 5'TCCACCACCCTGTTGCTGT-3' (reverse). The thermocycling conditions were: $96^{\circ} \mathrm{C}$ for $10 \mathrm{~min}, 45$ cycles of $96^{\circ} \mathrm{C}$ for $10 \mathrm{sec}, 60^{\circ} \mathrm{C}$ for $15 \mathrm{sec}$ and $72^{\circ} \mathrm{C}$ for $20 \mathrm{sec}$. The relative mRNA expression level was normalized to GAPDH and calculated using the $2^{-\Delta \Delta \mathrm{Ct}}$ method.

\section{Western Blot Analysis}

Cells were lysed in RIPA buffer on ice and centrifuged at 15,000 g for $15 \mathrm{~min}$. Protein concentration was measured using a BCA Protein Assay kit (Pierce, Rockford, IL, USA). An equal amount of protein was separated by $12 \%$ SDSPAGE and then transferred onto PVDF membranes (Millipore, Billerica, MA, USA). After blocking in 5\% nonfat milk, the membranes were incubated overnight at $4{ }^{\circ} \mathrm{C}$ with primary antibodies against PDIA6, Wnt3a, $\beta$-catenin and GAPDH. Subsequently, the membranes were washed three times with TBST and incubated with appropriate secondary antibody for $1 \mathrm{~h}$ at room temperature. All antibodies were purchased from Santa Cruz Biotechnology (Santa Cruz, CA, USA). Immunoreactive proteins were detected using an enhanced chemiluminescence detection system (Pierce, Rockford, IL, USA) and quantified by BandScan5.0.

\section{Cell Transfection}

Short hairpin RNA (shRNA) against PDIA6 (shPDIA6) and control shRNA (shNC) were synthesized by RiboBio (Guangzhou, China). The pcDNA3.1-PDIA6 expression vector and the empty vector were purchased from GeneCopoeia (Rockville, MD, USA). Cells were seeded in a 24-well plate and cultured for $24 \mathrm{~h}$. Subsequently, cells were transfected with shPDIA6 or pcDNA3.1-PDIA6 using Lipofectamine 3000 (Invitrogen, Carlsbad, CA, USA) according to the manufacturer's instructions. Fortyeight hours later, the knockdown or overexpression efficiency was confirmed by the RT-qPCR and Western blot analysis.

\section{CCK-8 Assay}

Cell proliferation was detected using the CCK-8 assay. After transfection, cells were seeded in a 96-well plate at a density of $2 \times 10^{3}$ cells/well and incubated for different times. Then, CCK-8 solution (Dojindo, Japan) was added to each well at different time points and cells were further incubated for $2 \mathrm{~h}$. The OD value was measured at $450 \mathrm{~nm}$ using a microplate reader. 
The cytotoxicity assay was used to evaluate the effect of cisplatin. After overnight incubation, transfected cells were treated with different concentrations of cisplatin and cultured for $48 \mathrm{~h}$. Subsequently, CCK-8 solution was added to each well. Cell viability was detected at $450 \mathrm{~nm}$ using a microplate reader. The survival of control cells was set at $100 \%$ and $\mathrm{IC}_{50}$ was calculated using CalcuSyn software.

\section{In vivo Xenograft Tumor Assay}

Female BALB/c nude mice, aged 4 to 5 weeks old, were obtained from Shanghai Laboratory Animal Center (Shanghai, China) and maintained under a specific pathogenfree condition in accordance with the National Institute of Health's Guide for the Care and Use of Laboratory Animals and was approved by the Animal Care and Use Committee of Qilu Hospital, Cheeloo College of Medicine. Transfected BGC-823 cells $\left(5 \times 10^{6}\right)$ were subcutaneously injected into left flank of mice. After palpable tumors were formed (tumor volume reached about $100 \mathrm{~mm}^{3}$ ), the mice were randomly divided into four groups $(\mathrm{n}=4)$ and intraperitoneally injected with or without cisplatin $(5 \mathrm{mg} / \mathrm{kg})$ twice a week. Tumor size was measured every week. Tumor volume was calculated by the following formula: $V=$ length $\times w_{i d t h}{ }^{2} \times 0.5$. Five weeks later, mice were euthanized and tumors were stripped and weighed.

\section{Statistical Analysis}

Data were shown as means \pm standard deviation (SD) and evaluated using SPSS 19.0 software. Comparison of different groups was performed using Student's $t$-test or one-way ANOVA. Graphs were generated using GraphPad Prism 5.0 software. $P<0.05$ was considered statistically significant.

\section{Results}

The Expression of PDIA6 is Increased in GC Tissues and Cell Lines

To elucidate the role of PDIA6 in GC, we first determined the expression of PDIA6 in GC tissues using RT-qPCR and Western blot analysis. The results showed that PDIA6 had a higher expression in GC tissues at both mRNA and protein levels than in corresponding normal tissues (Figure $1 \mathrm{~A}$ and $\mathrm{B})$. We also examined the expression levels of PDIA6 in GC cell lines (BGC-823 and MKN-45). We found that PDIA6 was overexpressed in BGC-823 and MKN-45 cell lines in comparison with the normal gastric epithelial cell line GES-1 (Figure 1C and D).

\section{Down-Regulation of PDIA6 Inhibits GC} Cell Proliferation

To further evaluate the specific effects of PDIA6 on cell proliferation, PDIA6 in GC cells was knocked down via shRNA transfection. The transfection efficiency was assessed by the RT-qPCR and Western blot analysis. The results showed that PDIA6 expression was significantly reduced in BGC-823 and MKN-45 cells at both mRNA and protein levels after shRNA transfection (Figure 2AD). Cell proliferation was measured using the CCK-8 assay. As shown in Figure 2E, the proliferative rate of BGC-823 cells was significantly reduced by PDIA6 knockdown in comparison with the control cells. We obtained similar results in MKN-45 cells (Figure 2F).

\section{Up-Regulation of PDIA6 Promotes GC Cell Proliferation}

To verify the tumor-promoting effect of PDIA6, the expression of PDIA6 was increased in GC cells via plasmid transfection. The overexpression efficiency was confirmed by the RT-qPCR and Western blot analysis. As expected, the mRNA and protein expression of PDIA6 were obviously upregulated in BGC-823 and MKN-45 cells after plasmid transfection (Figure 3AD). The CCK-8 assay was performed to evaluate cell proliferation. The results showed that PDIA6 up-regulation obviously accelerated the proliferation of BGC-823 and MKN-45 cells in comparison with corresponding control cells (Figure 3E and F).

\section{Down-Regulation of PDIA6 Inhibits Chemoresistance of GC Cells to Cisplatin} We also investigated the potential role of PDIA6 in chemoresistance of GC cells to cisplatin. The sensitivity of shPDIA6-transfected BGC-823 and MKN-45 cells to cisplatin was assayed $48 \mathrm{~h}$ after drug treatment. As shown in Figure 4A and B, knockdown of PDIA6 markedly enhanced the effect of cisplatin on BGC-823 cells and decreased the $\mathrm{IC}_{50}$ value of cisplatin in BGC-823 cells in comparison with the corresponding control cells. We obtained similar results in MKN-45 cells (Figure 4C and D).

\section{Up-Regulation of PDIA6 Promotes Chemoresistance of GC Cells to Cisplatin}

Then, we explored the effect of PDIA6 up-regulation on chemoresistance of GC cells to cisplatin. The sensitivity of PDIA6-overexpressed BGC-823 and MKN-45 cells to cisplatin was measured $48 \mathrm{~h}$ after drug treatment. The results 
A

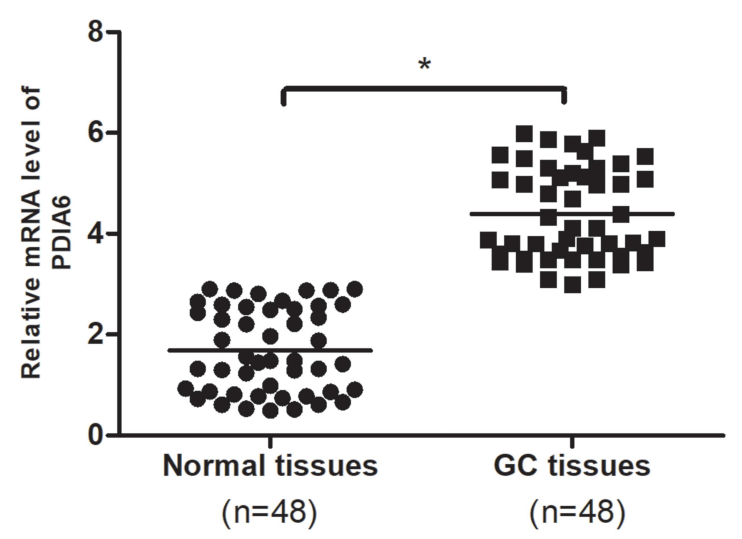

C

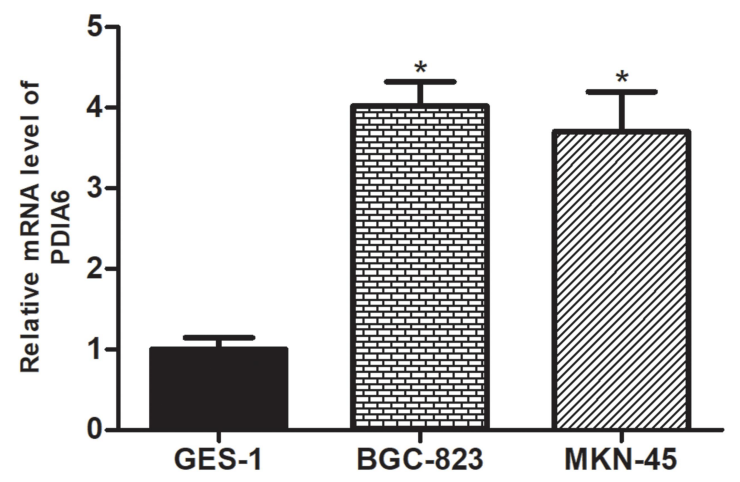

B

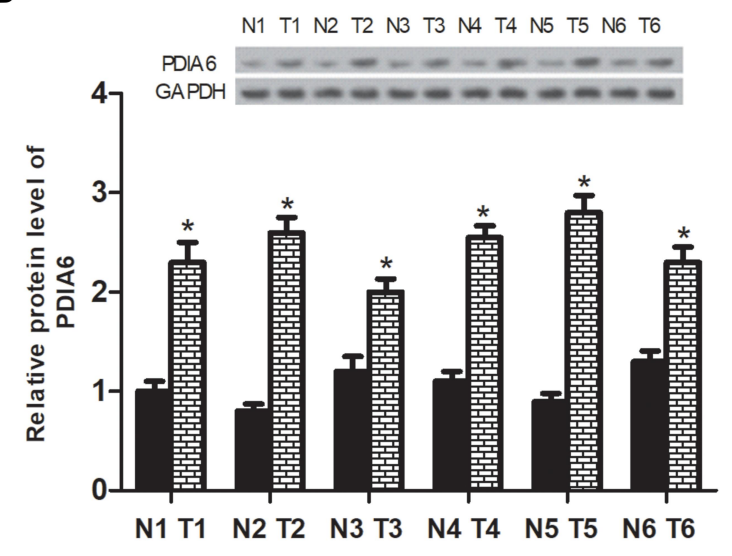

D

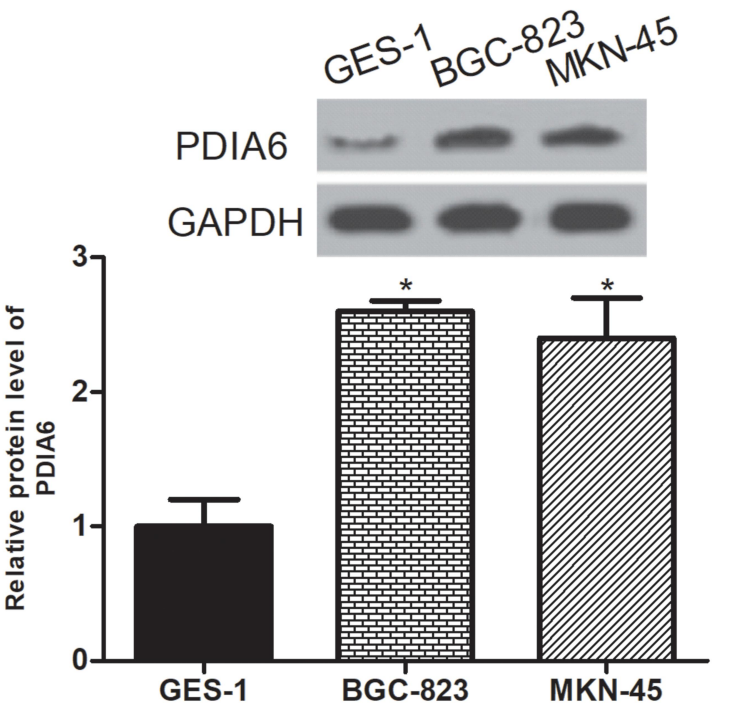

Figure I The expression of PDIA6 is increased in GC tissues and cell lines. (A and B) Relative PDIA6 mRNA and protein expression levels in GC tissues in comparison with matching normal tissues. $(n=48)$. ( $($ and $\mathbf{D})$ Relative PDIA6 mRNA and protein expression levels in GC cell lines (BGC-823 and MKN-45) in comparison with the normal gastric epithelial cell line GES-I. *p $<0.05$.

showed that PDIA6 up-regulation obviously blocked the effect of cisplatin on BGC-823 and MKN-45 cells and increased the $\mathrm{IC}_{50}$ value of cisplatin in these cells in comparison with corresponding control cells (Figure 5AD).

\section{PDIA6 Serves as a Regulator of the Wnt/ $\beta$-Catenin Signaling Pathway}

Previous studies have shown that the Wnt/ $\beta$-catenin signaling pathway plays an essential role in chemoresistance of GC cells, ${ }^{18,19}$ thus we examined whether this pathway was associated with the promoting effect of PDIA6 knockdown on chemosensitivity of GC cells to cisplatin. The results showed that the protein expression levels of Wnt3a and $\beta$-catenin were remarkably decreased by PDIA6 knockdown in BGC-
823 and MKN-45 cells (Figure 6A and B). In contrast, PDIA6 up-regulation increased the protein expression of Wnt3a and $\beta$-catenin in BGC-823 and MKN-45 cells (Figure 6C and D). These observations suggested PDIA6 as a regulator of the $\mathrm{Wnt} / \beta$-catenin signaling pathway.

\section{Wnt Activator Reverses the Inhibitory Effect of PDIA6 Knockdown on Cisplatin} Resistance in GC Cells

To further investigate the involvement of Wnt $/ \beta$-catenin pathway in PDIA6-regulated chemoresistance of GC cells, we used the Wnt pathway activator $\mathrm{LiCl}$ for the rescue experiments. The results showed that $\mathrm{LiCl}$ significantly restored the expression of Wnt3a and $\beta$-catenin in PDIA6-knockdown BGC-823 
A

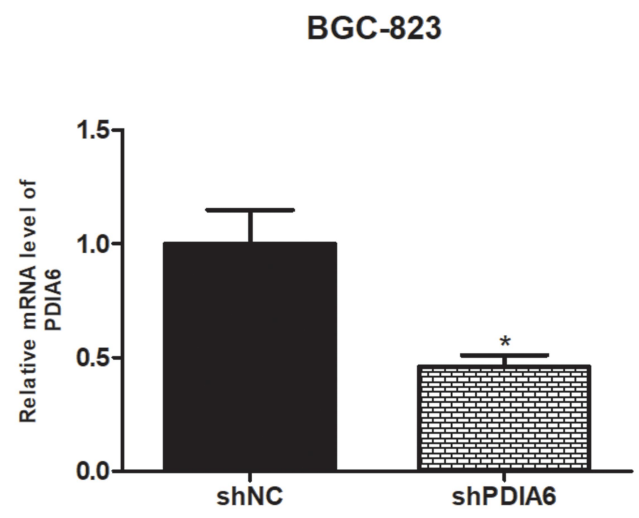

C

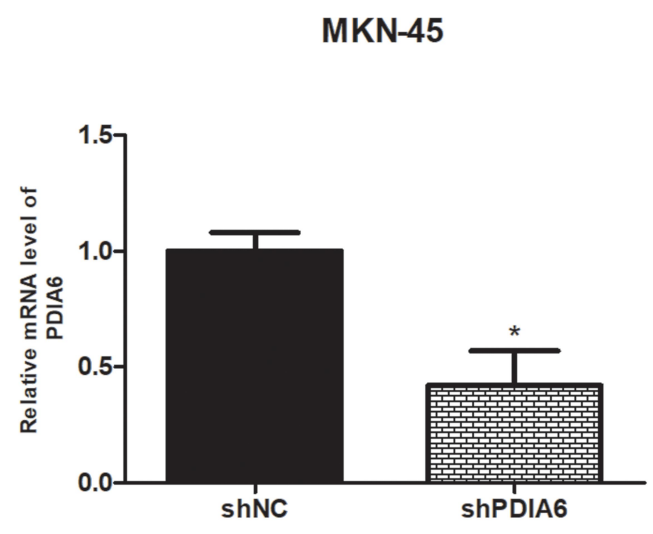

E

BGC-823

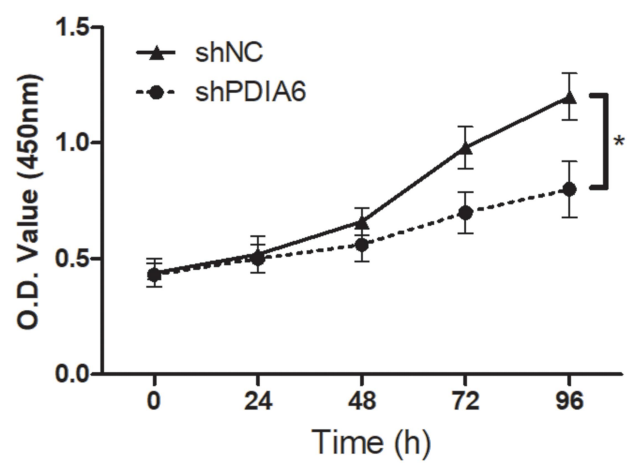

B

\section{BGC-823}

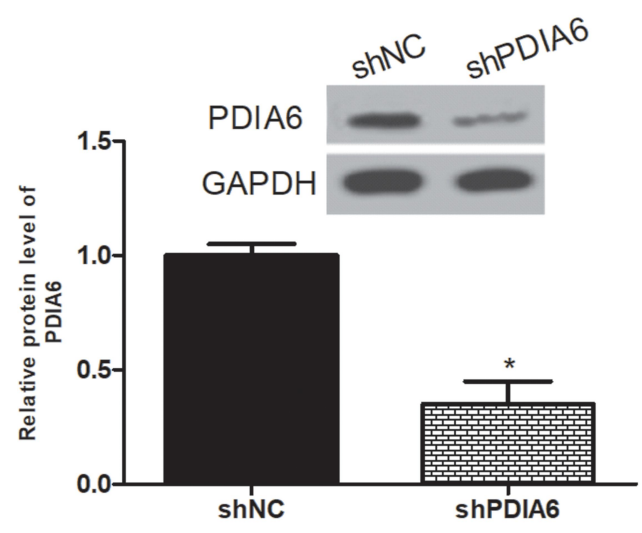

D

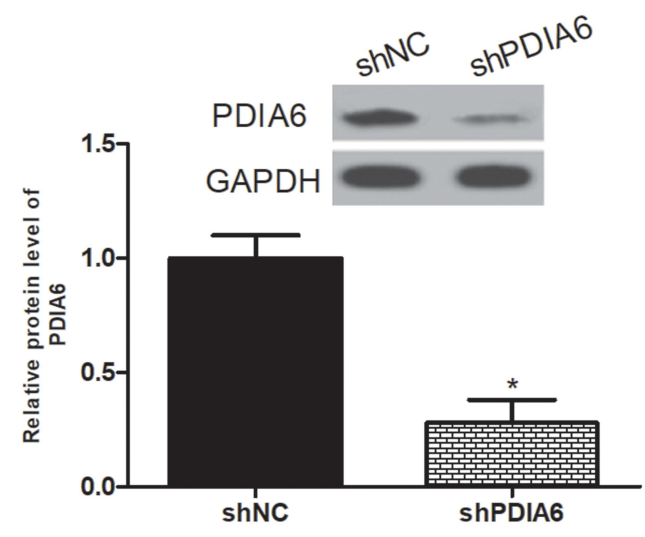

F

\section{MKN-45}

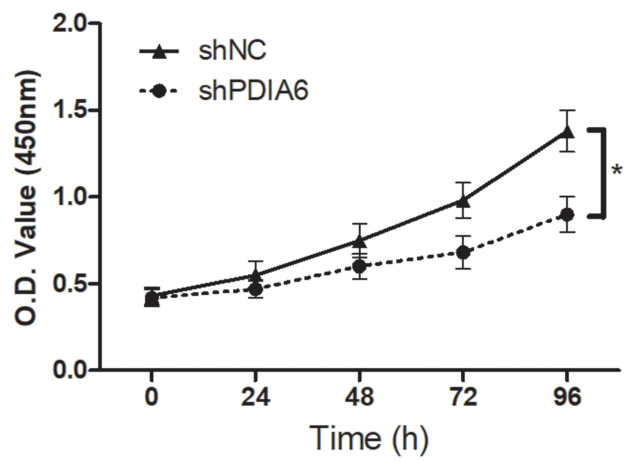

Figure 2 Down-regulation of PDIA6 inhibits GC cell proliferation. (A-D) PDIA6 knockdown in BGC-823 and MKN-45 cells was measured by the RT-qPCR and Western blot analysis after transfection. (E and F) BGC-823 and MKN-45 cell proliferation were measured using the CCK-8 assay. $* p<0.05$. 
A

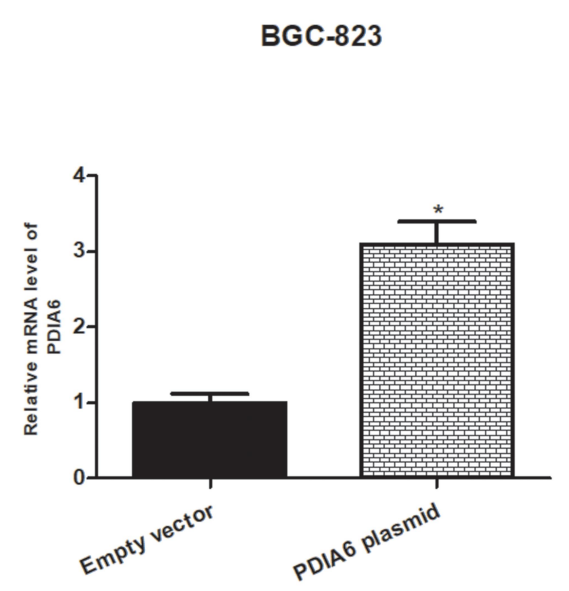

C

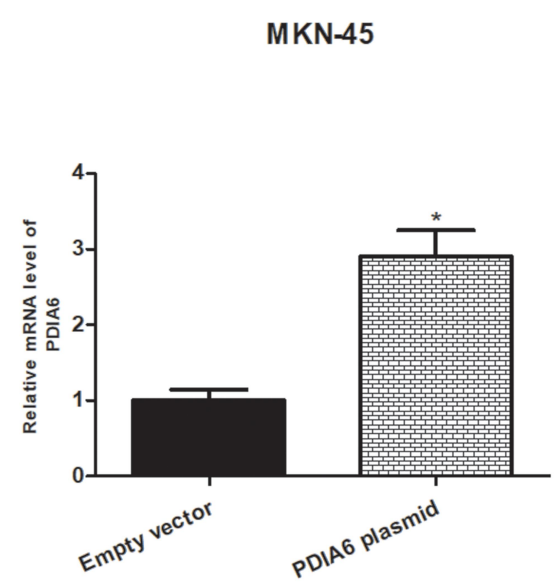

E

BGC-823

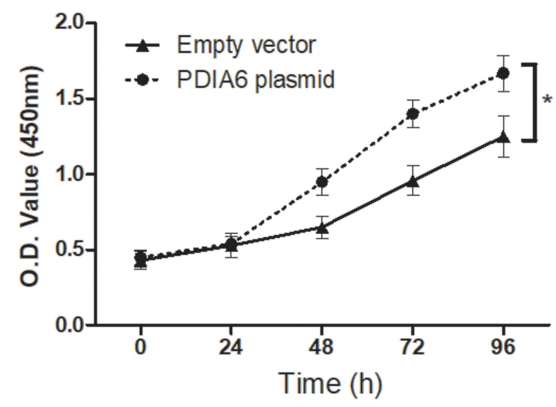

B

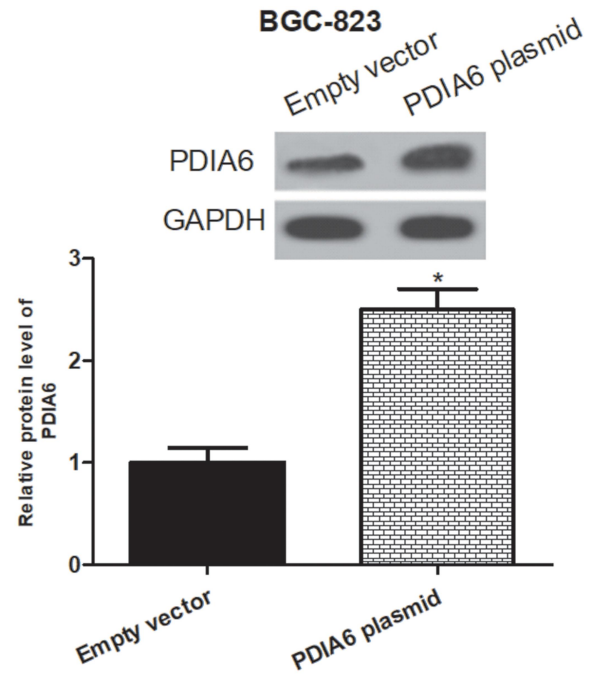

D

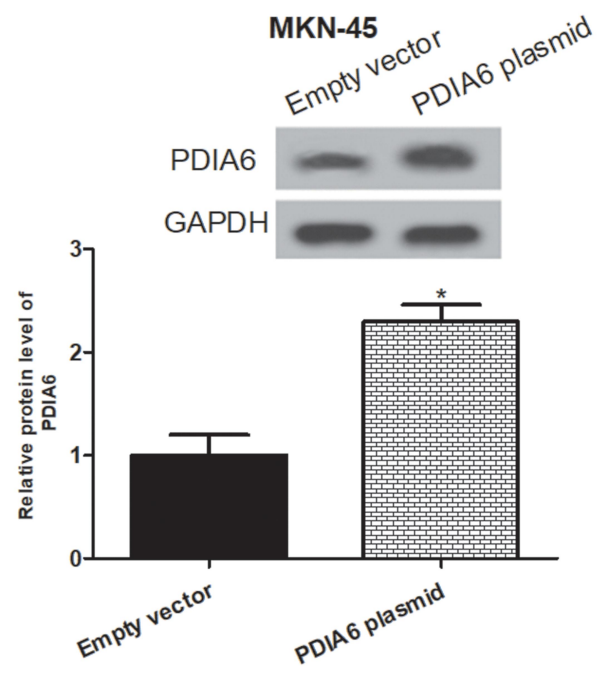

F

\section{MKN-45}

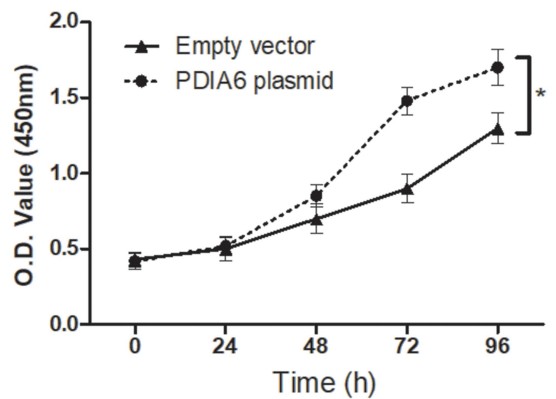

Figure 3 Up-regulation of PDIA6 promotes GC cell proliferation. (A-D) PDIA6 overexpression in BGC-823 and MKN-45 cells was verified by the RT-qPCR and Western blot analysis after transfection. (E and $\mathbf{F}$ ) The CCK-8 assay was performed to assess BGC-823 and MKN-45 cell proliferation. ${ }^{*} p<0.05$. 
A

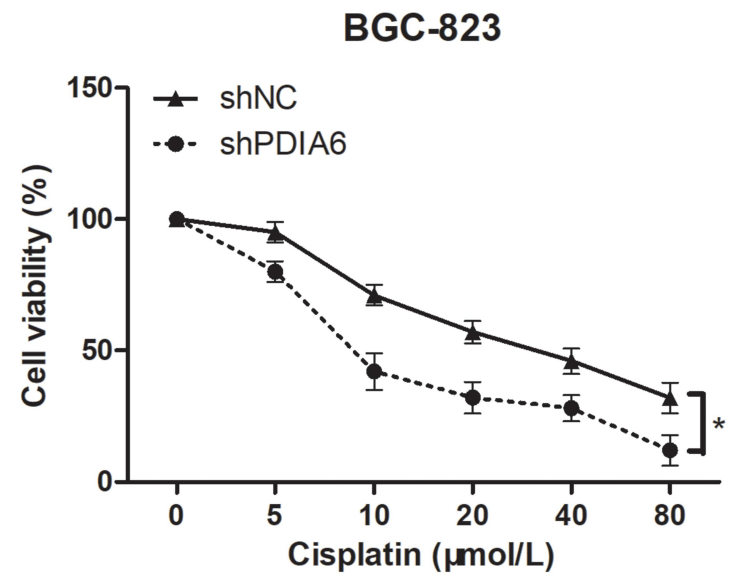

C

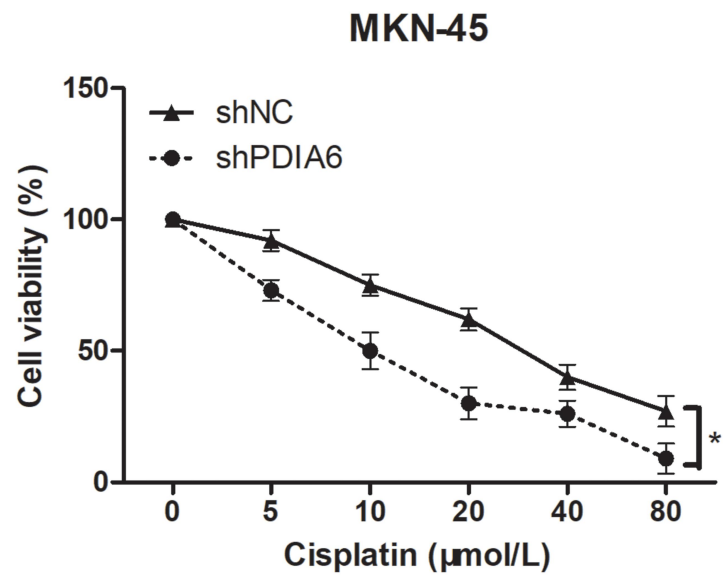

B

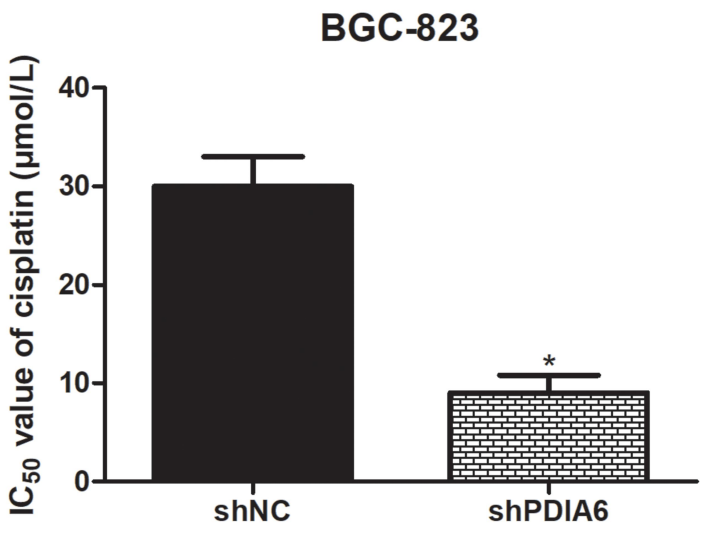

MKN-45

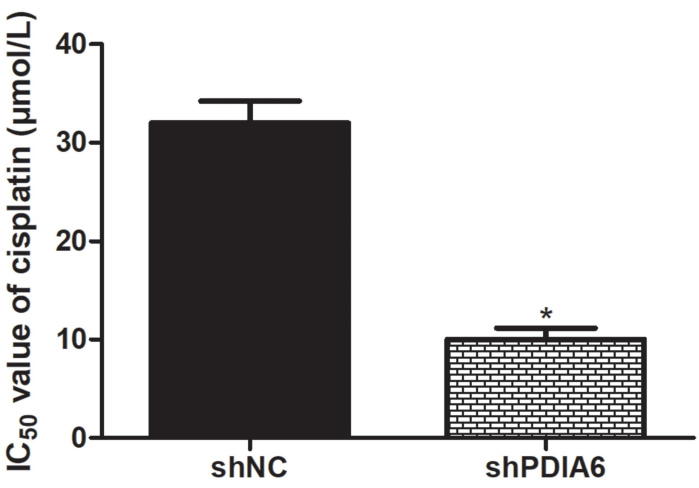

Figure 4 Down-regulation of PDIA6 inhibits chemoresistance of GC cells to cisplatin. After treatment with different concentration of cisplatin, the CCK-8 assay was used to measure BGC-823 (A and B) and MKN-45 (C and D) cell viability and sensitivity to cisplatin. * $p<0.05$.

and MKN-45 cells (Figure 7A and B). Furthermore, we found that $\mathrm{LiCl}$ partially reversed the inhibitory effect of PDIA6 knockdown on BGC-823 and MKN-45 cell proliferation (Figure 7C and D). We also observed that the suppressive effect of PDIA6 knockdown on cisplatin resistance in BGC823 and $\mathrm{MKN}-45$ cell was weakened by $\mathrm{LiCl}$ while the promoting effect of PDIA6 overexpression on cisplatin resistance was enhanced after $\mathrm{LiCl}$ treatment (Figure 7EH).

\section{PDIA6 Regulates the Chemosensitivity of GC Cells to Cisplatin in vivo}

To verify the tumor-promoting effect of PDIA6 in vivo, the xenograft tumor assay was performed. BGC-823 cells transfected with shPDIA6 or shNC were subcutaneously injected into nude mice. After formation of palpable tumors, the mice were treated with or without cisplatin. Tumor size was measured every week. Five weeks later, the mice were euthanized and tumors were stripped and weighed. As shown in Figure 8A, PDIA6 knockdown significantly inhibited tumor growth and meanwhile potentiated the suppressive effect of cisplatin. Consistently, PDIA6 downregulation markedly reduced tumor weight and simultaneously enhanced the effect of cisplatin (Figure 8B).

\section{Discussion}

Accounting for a high morbidity and mortality in the world, GC remains a focus of public health issues. ${ }^{20}$ The absence of specific symptoms makes early diagnosis of 


\section{A}

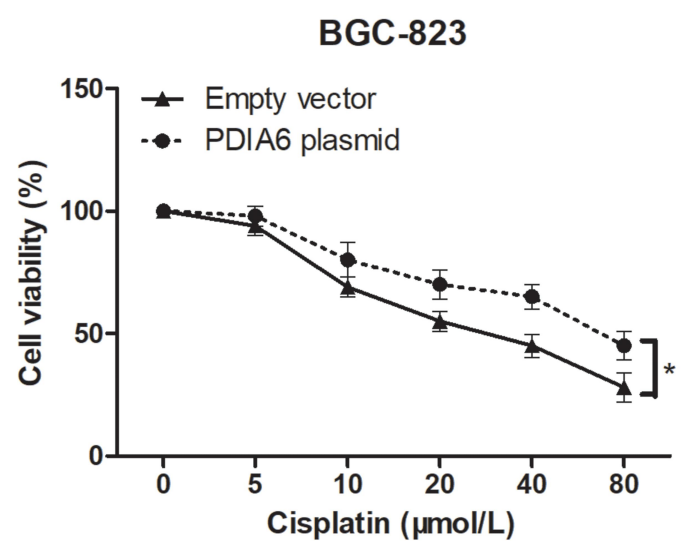

C

MKN-45

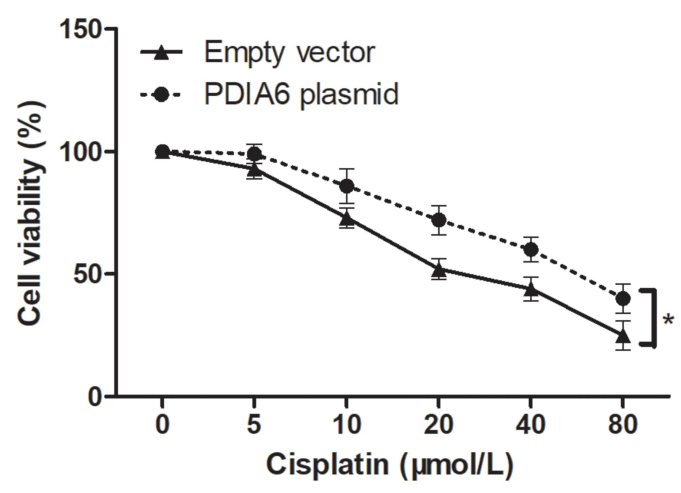

B

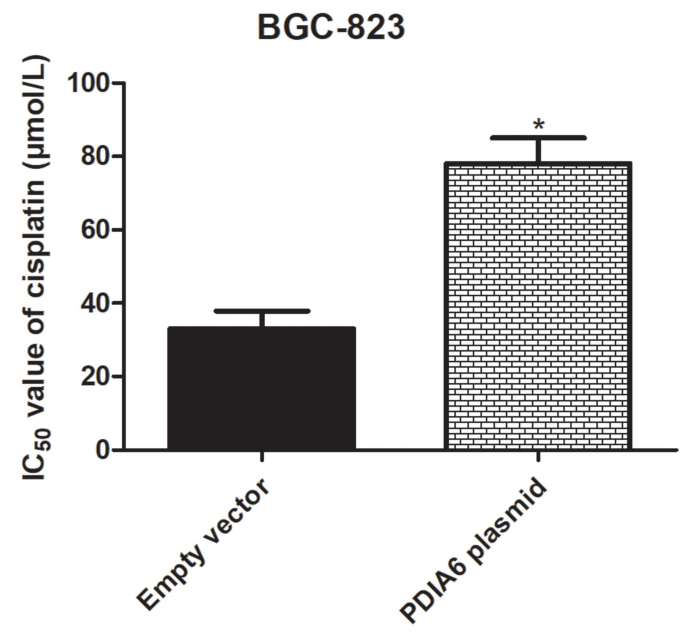

D

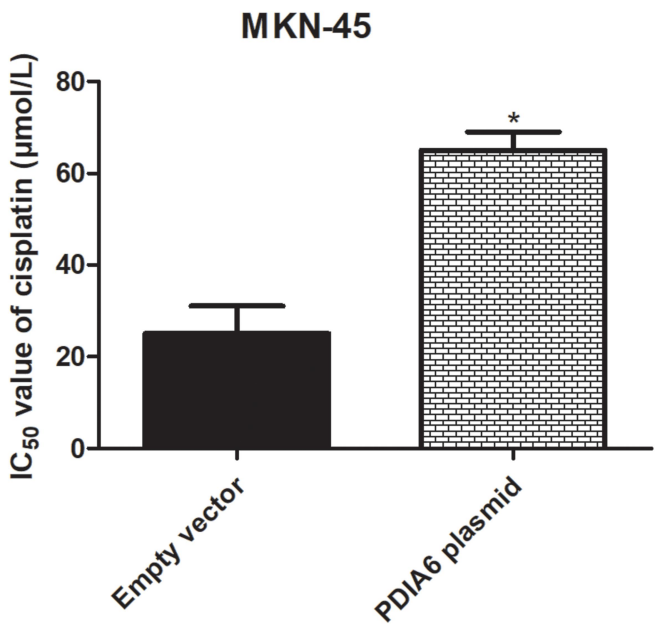

Figure 5 Up-regulation of PDIA6 promotes chemoresistance of GC cells to cisplatin. BGC-823 (A and B) and MKN-45 (C and D) cells were treated with different concentration of cisplatin, followed by detection of cell viability and sensitivity to cisplatin. ${ }^{*} p<0.05$.

GC difficult, generally resulting in a poor prognosis. ${ }^{21,22}$ This circumstance is worsened by development of chemotherapeutic resistance. ${ }^{7}$ Therefore, elucidation of the molecular mechanism underlying chemoresistance in GC will deliver significant benefits to GC treatment.

PDIA6, a member of the PDI family, is ubiquitously expressed in diverse human tissues and could be induced under ischemic and hypoxic stress. ${ }^{23}$ Recently, PDIA6 has been reported to be closely associated with progression of various cancers. For example, Ramos et al demonstrated overexpression of PDIA6 in breast cancer and suggested PDIA6 as a prognostic marker of aggressiveness. ${ }^{24}$ In this study, we found that the expression of PDIA6 was elevated in GC tissues and cell lines. In addition, knockdown of PDIA6 inhibited GC cell proliferation in vitro and in vivo. Like us, Gao et al reported that overexpression of PDIA6 increased the proliferative ability of cervical cancer cells accompanied with accelerated cell cycle progression. ${ }^{16}$ These observations proposed the role of PDIA6 as a tumor promoter in cancer progression.

As a first-line chemotherapeutic agent, cisplatin could cause DNA damage to eliminate proliferating cancer cells. ${ }^{25-27}$ But this anti-cancer effect is frequently 
A

BGC-823

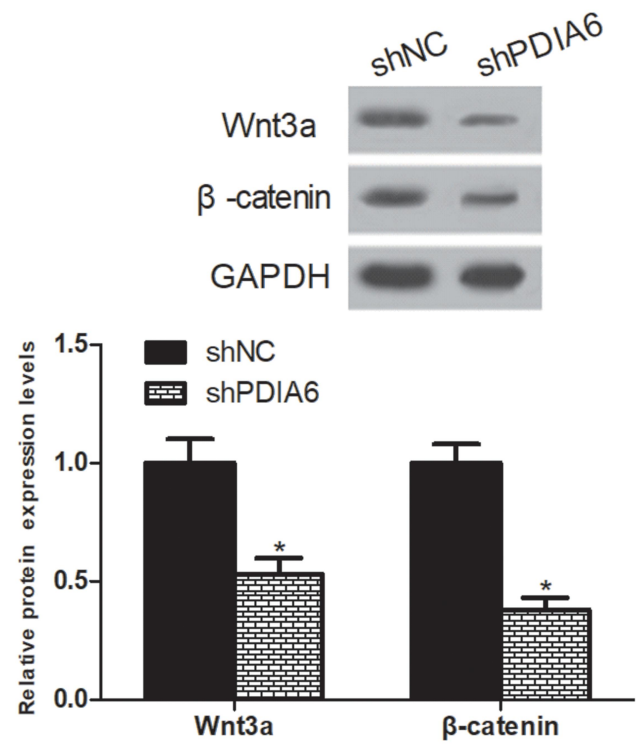

C

BGC-823

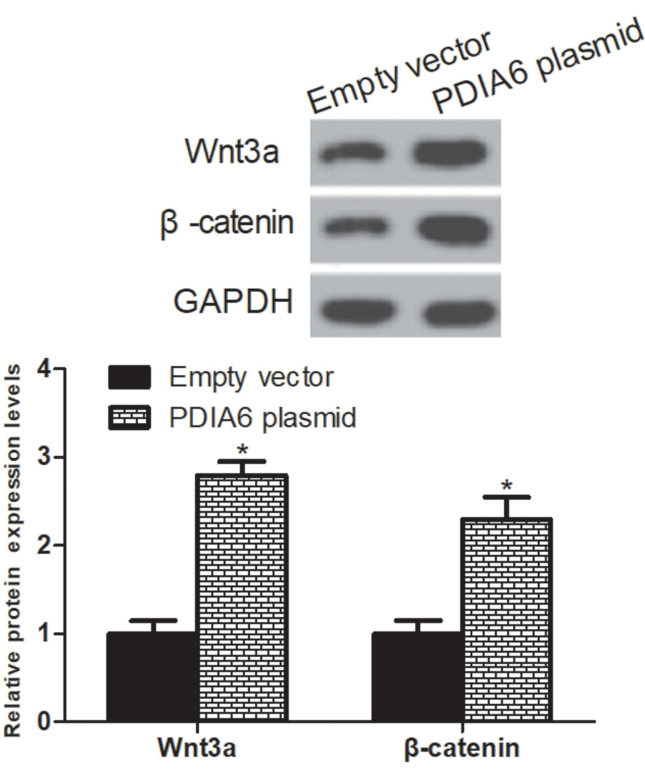

B

\section{MKN-45}

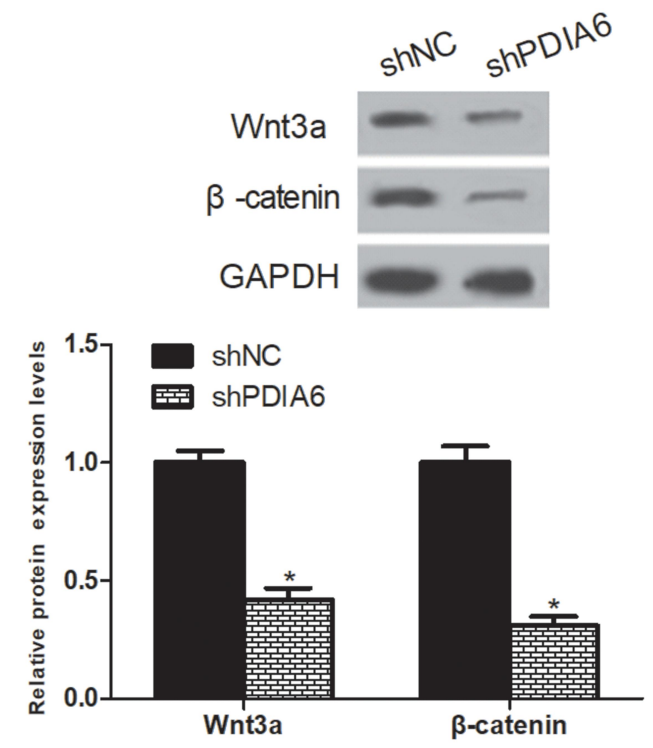

D

MKN-45
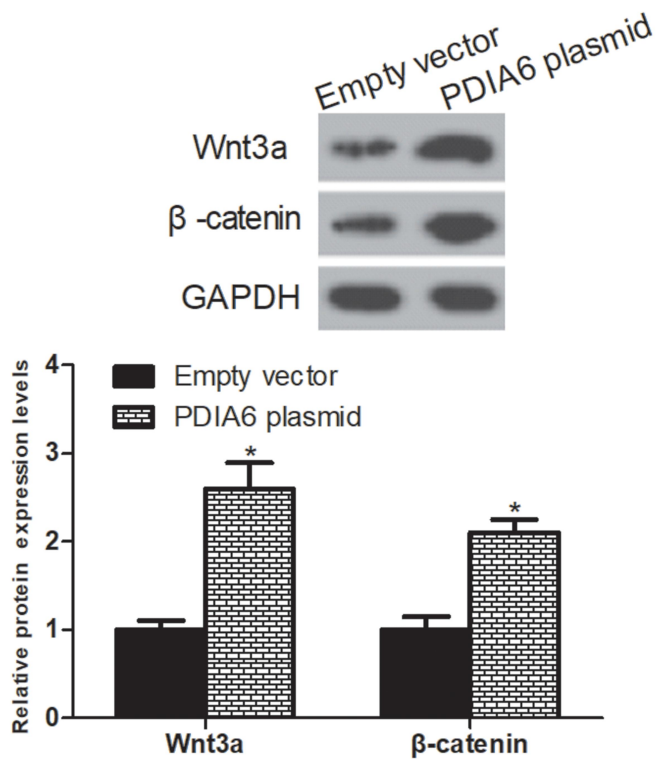

Figure 6 PDIA6 serves as a regulator of the $\mathrm{Wnt} / \beta$-catenin signaling pathway. The protein expression of $\mathrm{Wnt} 3 \mathrm{a}$ and $\beta$-catenin was detected by the $\mathrm{Western}$ blot analysis, after PDIA6 was knockdown (A and B) or overexpressed (C and D) in BGC-823 and MKN-45 cells. ${ }^{*} p<0.05$.

limited by development of chemoresistance, finally resulting in therapeutic failure. ${ }^{28-30}$ A previous study reported that PDIA6 could regulate the chemosensitivity to cisplatin in lung adenocarcinoma cells, ${ }^{17}$ suggesting that PDIA6 may have an association with chemoresistance. In this study, we further examined the correlation between PDIA6 expression and chemosensitivity to cisplatin in GC cells in vitro and in vivo. The results showed that the $\mathrm{IC}_{50}$ value of cisplatin in GC cells was markedly decreased by PDIA6 
A

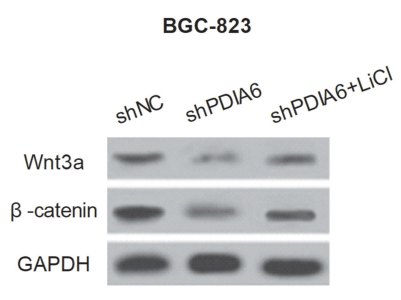

B

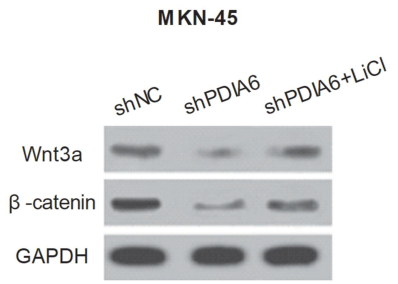

F

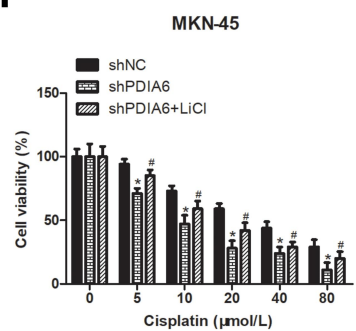

E

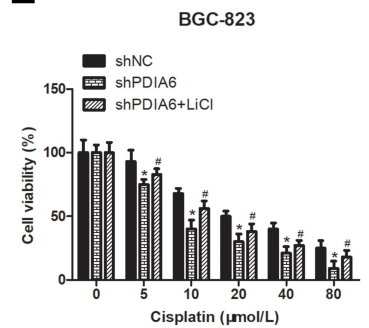

C

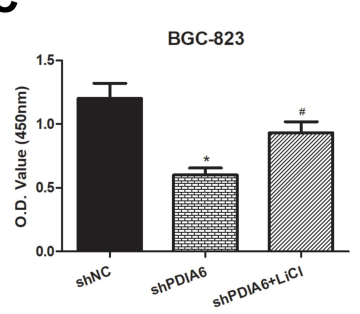

D

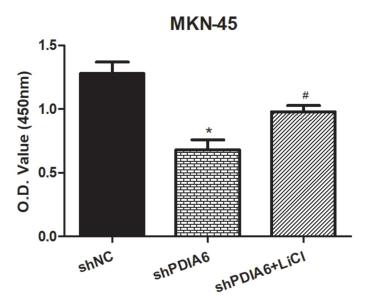

G

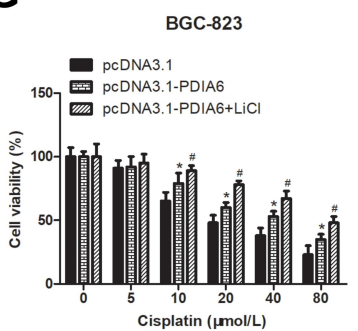

H

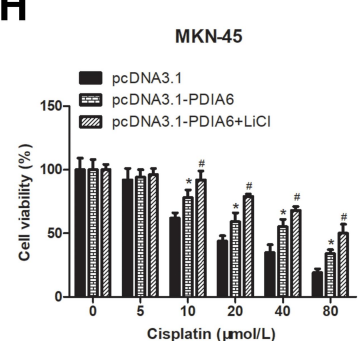

Figure 7 Wnt activator reverses the inhibitory effect of PDIA6 knockdown on cisplatin resistance in GC cells. BGC-823 and MKN-45 cells were transfected with shNC or shPDIA6 for $48 \mathrm{~h}$ in the presence of $\mathrm{LiCl}(20 \mathrm{mmol} / \mathrm{L})$. (A and B) The protein expression of Wnt3a and $\beta$-catenin was detected by the Western blot analysis. (C-F) Cell viability was determined using the CCK-8 assay in the presence or absence of cisplatin. ${ }^{*} p<0.05$ vs shNC; ${ }_{p}<0.05$ vs shPDIA6. BGC-823 and MKN-45 cells were transfected with pcDNA3.I or pcDNA3.I-PDIA6 for $48 \mathrm{~h}$ in the presence of $\mathrm{LiCl}(20 \mathrm{mmol} / \mathrm{L})$. ( $\mathbf{G}$ and $\mathbf{H})$ Cell viability was measured by the CCK-8 assay in the presence or absence of cisplatin. ${ }^{*} p<0.05$ vs pcDNA3.I; ${ }^{\#} p<0.05$ vs pcDNA3.I-PDIA6.

A
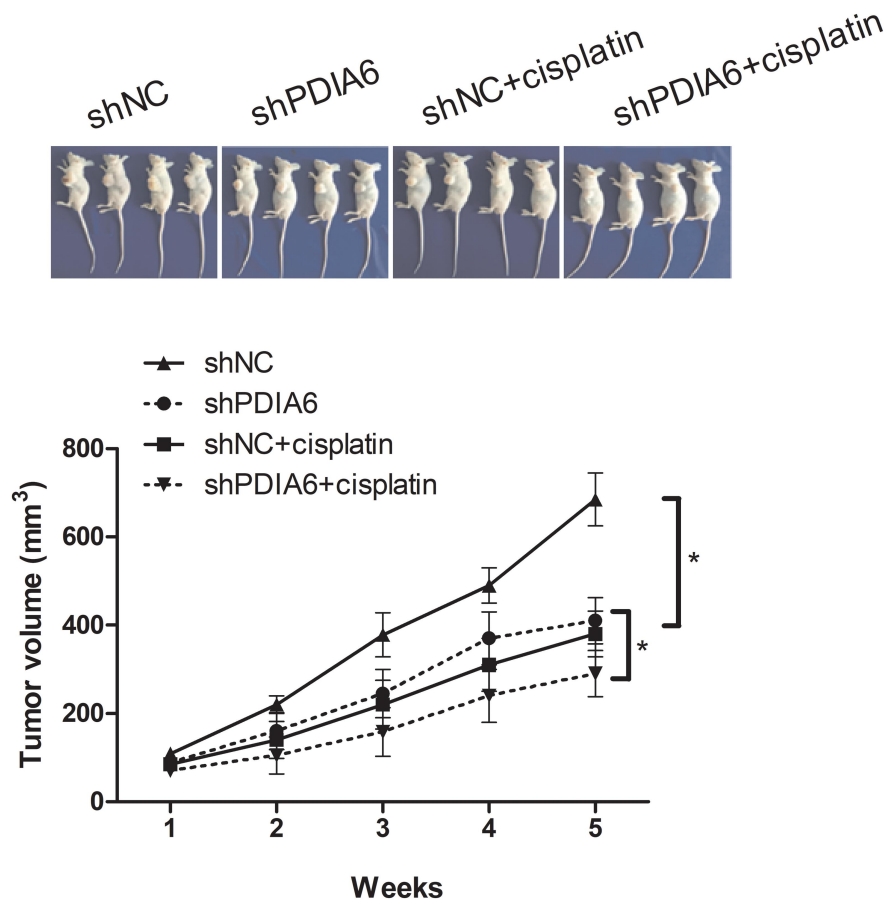

B
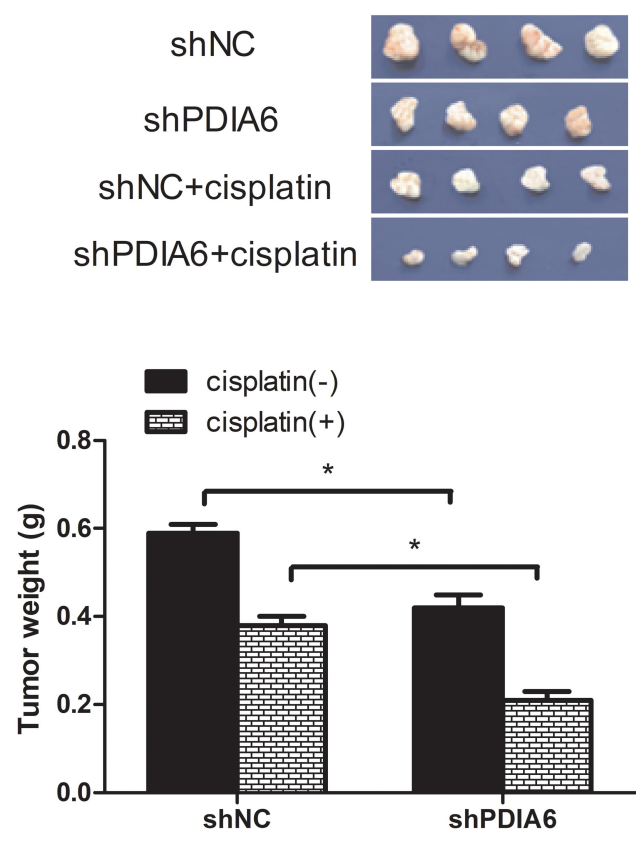

Figure 8 PDIA6 regulates GC cell proliferation and chemoresistance in vivo. Mice were injected with shPDIA6- or shNC-transfected BGC-823 cells and then treated with or without cisplatin after formation of palpable tumors. Tumor volume (A) was observed every week and tumor weight (B) was measured after five weeks of injection. ${ }^{*} p<0.05$. 
knockdown. Furthermore, the sensitivity to cisplatin was significantly enhanced after PDIA6 knockdown in GC cells.

The Wnt/ $\beta$-catenin signaling pathway is known as an important regulator of various crucial cellular processes. $^{31,32}$ Moreover, the pathway has been demonstrated to play an essential role in chemoresistance of GC cells. $^{18,19}$ Notably, PDIA6 was found interacting with the Wnt/ $\beta$-catenin pathway to affect cancer progression. ${ }^{16}$ In this study, we showed that PDIA6 knockdown remarkably decreased the protein expression of $\mathrm{Wnt} 3 \mathrm{a}$ and $\beta$-catenin in GC cells. Furthermore, we used the Wnt pathway activator $\mathrm{LiCl}$ to further investigate the involvement of $\mathrm{Wnt} / \beta$-catenin pathway in PDIA6-regulated chemoresistance of GC cells. The results showed that $\mathrm{LiCl}$ significantly restored the expression of $\beta$-catenin in PDIA6-knockdown GC cells and meanwhile reversed the inhibitory effect of PDIA6 knockdown on cisplatin resistance in GC cells.

\section{Conclusion}

This study revealed that PDIA6 was overexpressed in GC tissues and cell lines. Down-regulation of PDIA6 inhibited GC cell proliferation and chemoresistance to cisplatin while up-regulation of PDIA6 promoted the proliferation and chemoresistance of GC cells. Besides, PDIA6 regulated the chemosensitivity of GC cells to cisplatin in vivo. Mechanically, PDIA6 served as a regulator of the Wnt/ $\beta$ catenin signaling pathway by affecting the protein expression of Wnt3a and $\beta$-catenin and Wnt activator reversed the inhibitory effect of PDIA6 knockdown on cisplatin resistance in GC cells. These findings provided new insight into biological functions of PDIA6 and suggested it as a potential target for drug resistance in GC chemotherapy.

\section{Disclosure}

The authors declare that they have no conflicts of interest.

\section{References}

1. Ferlay J, Soerjomataram I, Dikshit R, et al. Cancer incidence and mortality worldwide: sources, methods and major patterns in GLOBOCAN 2012. Int $J$ Cancer. 2015;136(5):E359E386. doi:10. 1002/ijc. 29210

2. Song Z, Wu Y, Yang J, Yang D, Fang X. Progress in the treatment of advanced gastric cancer. Tumor Biol. 2017;39(7):1010428317714626. doi:10.1177/1010428317714626

3. Smyth EC, Cunningham D. Targeted therapy for gastric cancer. Curr Treat Options Oncol. 2012;13(3):377-389. doi:10.1007/s11864-0120192-6

4. Mayer RJ, Venook AP, Schilsky RL. Progress against GI cancer during the American Society of Clinical Oncology's first 50 years. J Clin Oncol. 2014;32(15):1521-1530. doi:10.1200/JCO.2014.55.4121
5. Janunger KG, Hafström L, Glimelius B. Chemotherapy in gastric cancer: A review and updated meta-analysis. Eur J Surg. 2014;168 (11):597-608. doi:10.1080/11024150201680005

6. Lordick F, Lorenzen S, Yamada Y, Ilson D. Optimal chemotherapy for advanced gastric cancer: is there a global consensus? Gastric Cancer. 2014;17(2):213-225. doi:10.1007/s10120-013-0297-z

7. Gillet JP, Gottesman MM. Mechanisms of multidrug resistance in cancer. Methods Mol Biol. 2010;596(596):47-76.

8. Wang L, Wang X, Wang CC. Protein disulfide-isomerase, a folding catalyst and a redox-regulated chaperone. Free Radic Biol Med. 2015;83:305-313. doi:10.1016/j.freeradbiomed.2015.02.007

9. Galligan JJ, Petersen DR. The human protein disulfide isomerase gene family. Hum Genomics. 2012;6(1):6. doi:10.1186/1479-7364-6-6

10. Sato Y, Kojima R, Okumura M, et al. Synergistic cooperation of PDI family members in peroxiredoxin 4-driven oxidative protein folding. Sci Rep. 2013;3:2456. doi:10.1038/srep02456

11. Kwok CT, Morris AG, Frampton J, Smith B, Shaw CE, De Belleroche J. Association studies indicate that protein disulfide isomerase is a risk factor in amyotrophic lateral sclerosis. Free Radic Biol Med. 2013;58:81-86. doi:10.1016/j.freeradbiomed.2013.01.001

12. Halloran M, Parakh S, Atkin JD. The role of s-nitrosylation and s-glutathionylation of protein disulphide isomerase in protein misfolding and neurodegeneration. Int J Cell Biol. 2013;2013:1-15. doi:10.1155/2013/797914

13. Parakh S, Atkin JD. Novel roles for protein disulphide isomerase in disease states: a double edged sword? Front Cell Dev Biol. 2015;3 (30):30. doi:10.3389/fcell.2015.00030

14. Andreu CI, Woehlbier U, Torres M, Hetz C. Protein disulfide isomerases in neurodegeneration: from disease mechanisms to biomedical applications. FEBS Lett. 2012;586(18):2826-2834. doi:10.1016/j. febslet.2012.07.023

15. Benham AM. The protein disulfide isomerase family: key players in health and disease. Antioxid Redox Signal. 2012;16(8):781-789. doi:10.1089/ars.2011.4439

16. Gao H, Sun B, Fu H, et al. PDIA6 promotes the proliferation of HeLa cells through activating the Wnt/ $\beta$-catenin signaling pathway. Oncotarget. 2016;7(33):53289-53298. doi:10.18632/oncotarget.10795

17. Tufo G, Jones AWE, Wang Z, et al. The protein disulfide isomerases PDIA4 and PDIA6 mediate resistance to cisplatin-induced cell death in lung adenocarcinoma. Cell Death Differ. 2014;21(5):685-695. doi:10.1038/cdd.2013.193

18. Huang H, Chen Z, Ni X. Tissue transglutaminase-1 promotes stemness and chemoresistance in gastric cancer cells by regulating Wnt/ $\beta$ catenin signaling. Exp Biol Med. 2016;242(2):194-202. doi:10.1177/ 1535370216670541

19. Cai W, Chen G, Luo Q, et al. PMP22 regulates self-renewal and chemoresistance of gastric cancer cells. Mol Cancer Ther. 2017;16 (6):1187-1198. doi:10.1158/1535-7163.MCT-16-0750

20. Mclean MH, Elomar EM. Genetics of gastric cancer. Nat Rev Gastroenterol Hepatol. 2014;11(11):664-674. doi:10.1038/nrgastro. 2014.143

21. Montenegro RC, Clark PG, Howarth A, et al. BET inhibition as a new strategy for the treatment of gastric cancer. Oncotarget. 2016;7(28):43997-44012. doi:10.18632/oncotarget.9766

22. Bo S, Jin Y, Chao L, Zhou H, Zheng Y. Tumor suppressor role of miR-363-3p in gastric cancer. Med Sci Monit. 2015;21:4074-4080. doi:10.12659/MSM.896556

23. Vekich JA, Belmont PJ, Thuerauf DJ, Glembotski CC. Protein disulfide isomerase-associated 6 is an ATF6-inducible ER stress response protein that protects cardiac myocytes from ischemia/reperfusion-mediated cell death. J Mol Cell Cardiol. 2012;53(2):259-267. doi:10.1016/j.yjmcc.2012.05.005

24. Ramos FS, Serino LTR, Carvalho CMS, et al. PDIA3 and PDIA6 gene expression as an aggressiveness marker in primary ductal breast cancer. Genet Mol Res. 2015;14(2):6960-6967. doi:10.4238/2015. June. 26.4 
25. Xu S, Huang $\mathrm{H}$, Chen $\mathrm{YN}$, et al. DNA damage responsive miR-33b$3 \mathrm{p}$ promoted lung cancer cells survival and cisplatin resistance by targeting p21WAF1/CIP1. Cell Cycle. 2016;15(21):2920-2930. doi:10.1080/15384101.2016.1224043

26. Seah KS, Loh JY, Trang TN, et al. SAHA and cisplatin sensitize gastric cancer cells to doxorubicin by induction of DNA damage, apoptosis and perturbation of AMPK-mTOR signalling. Exp Cell Res. 2018;370(2):283-291.

27. Chen D, Lin X, Gao J, et al. Wee1 inhibitor AZD1775 combined with cisplatin potentiates anticancer activity against gastric cancer by increasing DNA damage and cell apoptosis. Biomed Res Int. 2018;2018:5813292.

28. Necchi A, Pond GR, Giannatempo P, et al. Cisplatin-based first-line therapy for advanced urothelial carcinoma after previous perioperative cisplatin-based therapy. Clin Genitourin Cancer. 2015;13 (2):178-184. doi:10.1016/j.clgc.2014.08.010
29. Dasari S, Tchounwou PB. Cisplatin in cancer therapy: molecular mechanisms of action. Eur J Pharmacol. 2014;740:364-378. doi:10.1016/j.ejphar.2014.07.025

30. Huang J, Zhao Y, Xu Y, et al. Comparative effectiveness and safety between oxaliplatin-based and cisplatin-based therapy in advanced gastric cancer: A meta-analysis of randomized controlled trials. Oncotarget. 2016;7(23):34824-34831. doi:10.18632/oncotarget.9189

31. Matsuzaki S, Darcha C. Involvement of the Wnt/ $\beta$-catenin signaling pathway in the cellular and molecular mechanisms of fibrosis in endometriosis. PLoS One. 2013;8(10):e76808. doi:10.1371/journal. pone. 0076808

32. Jiang J, Shi S, Zhou Q, et al. Downregulation of the Wnt/ $\beta$-catenin signaling pathway is involved in manganese-induced neurotoxicity in rat striatum and PC12 cells. J Neurosci Res. 2014;92(6):783-794.

\section{Publish your work in this journal}

Cancer Management and Research is an international, peer-reviewed open access journal focusing on cancer research and the optimal use of preventative and integrated treatment interventions to achieve improved outcomes, enhanced survival and quality of life for the cancer patient.
The manuscript management system is completely online and includes a very quick and fair peer-review system, which is all easy to use. Visit http://www.dovepress.com/testimonials.php to read real quotes from published authors. 